\title{
Fine Tuning of the Longitudinal Plasmon Resonance of Gold Nanorods by Depleting Gold Precursor
}

\author{
Yingying Wang, ${ }^{\dagger}$ Saran Long, ${ }^{\dagger}$ Silvije Vdovic, ${ }^{\dagger, \dagger}$ and Xuefei Wang ${ }^{*}{ }^{\dagger}$ \\ ${ }^{\dagger}$ Beijing National Laboratory for Molecular Sciences, Institute of Chemistry, Chinese Academy of Sciences, Beijing 100190, China \\ ${ }^{\ddagger}$ Institute of Physics, Bijenička cesta 46, Zagreb 10000, Croatia
}

Supporting Information

ABSTRACT: For gold nanorod synthesis, the seed-mediated growth as the most effective method has been used widely. However, the inevitable overgrowth leads to poor control of the final shape of particle. In present work, by adding chemical inhibitor to deplete the residual gold salt in solution, the growth process can be terminated effectively at any stage and nanorods with desirable shape can be produced. This kind of competitive precursor depletion provides a simple pathway to finely tune the wavelength of the longutidinal plasmon band of gold nanorods. It also has potential application in studying the crystallization mechanism of nonspherical metal nanoparticles in solution.

KEYWORDS: gold nanorods, shape control, depletion, longitudinal plasmon band, aspect ratio

\section{INTRODUCTION}

Production of gold nanocrystals with well-defined shapes appears to be one of the most popular subjects of research over the past decade, as judged from the total number of publications per year on this subject. ${ }^{1,2}$ Until now numerous gold nanoparticles with various shapes have been synthesized and studied. Among them, gold nanorods (NRs) have attracted the most attention and have been widely investigated, because of the tunable longitudinal plasmon resonance (LPR) that offers gold NRs many unique properties and potential applications such as in photothermal therapy, ${ }^{3,4}$ photodynamic therapy, $^{5-8}$ cell labeling ${ }^{9,10}$ and optical filter design. ${ }^{11}$ Unlike spherical particle, gold NRs have two plasmon resonance bands. One, constantly around $520 \mathrm{~nm}$, is assigned to the transverse mode parallel to the short axis. The other, i.e. LPR, is contributed from longitudinal mode along the long axis characterized as variable absorption band ranging from visible to near-infrared region mainly dependent on the aspect ratio (AR) of rods. Therefore, for the synthesis of gold NRs, fine control over the AR should be one of the most important, yet a challenging task.

Many methods have been developed for gold NR synthesis, such as template growth, ${ }^{12}$ photochemical synthesis, ${ }^{13}$ seedmediated growth in solution ${ }^{12,14}$ and electron-beam lithography. ${ }^{15}$ Among them, the seed-mediated method developeded by El-Sayed and Murphy, ${ }^{12,14}$ as the simplest one to produce gold NRs in high quantity, has been widely used. Although, by varying the concentration of silver nitrate in growth solution, NRs of varied aspect ratio could be synthesized, further study suggested that the dependence of AR on the silver nitrate amount is rather complex. ${ }^{16}$ Morphology of nanorods is affected by many factors, ${ }^{2}$ such as temperature, ${ }^{17,18}$ surfactant type ${ }^{18}$ concentration of gold salt, reducing agent, seed and silver nitrate, ${ }^{19}$ seed quality, ${ }^{20} \mathrm{pH}^{21}$ even stirring. ${ }^{22}$ Considering the dependence of the photophysical properties on morphology, it is critical to develop tunable synthesis of gold NRs.

To synthesize gold NRs with desired LPR, Wang and coworkers have developed the chemically end-etching approach, ${ }^{23,24}$ where the as-synthesized NRs were gradually shortened by oxidative etching occurring preferentially at the both ends. More importantly, this etching process was strongly $\mathrm{pH}$ and temperature dependent, and the LPR could be tuned accurately via real-time monitoring of the absorption spectrum. To the best of our knowledge, this is the most effective approach available for AR tuning developed so far, where the real-time measurement of the absorption spectrum has been demonstrated as a powerful tool in controlling AR for gold NRs synthesis. Real-time absorption spectrum has also been employed in investigating the volume increase, aspect ratio change and shape evolution to obtain the growth dynamics of gold NRs in solution. ${ }^{16,19,21,25,26}$

It is worth noting that both in our experiments and other works concerning the gold NRs, ${ }^{19,21,25,26}$ a common evolution trend has been observed in the real-time absorption spectra: following seed addition the LPR appeared and then underwent a red shift within the first several minutes, followed by a gradual blue shift that continued over $30 \mathrm{~min}$. As the longitudinal plasmon band central wavelength is linearly proportional to the

Special Issue: Synthetic and Mechanistic Advances in Nanocrystal Growth

Received: June 13, 2012

Revised: August 30, 2012

Published: October 9, 2012 
aspect ratio of gold $\mathrm{NRs}^{27}$ the blue shift observed here indicates that the formation of the gold NRs in solution is not a simple process where the volume increases monotonically, but involves a shape adjustment, i.e., an AR decrease. Obviously, the uncontrollable $\mathrm{AR}$ reduction here results in an uncertain LPR wavelength for the final NRs. There are two explanations acounting for the AR decrease: First, at the end of the growth process, all Au precursors have deposited on particles, but the atom diffusion from the end facet to side facet caused by the minimization of the total interfacial free energy leads to AR decrease; Second, at the later growth stage the deposition selectivity becomes weak and the same growth rate on the side and end facets of NRs results in AR decrease. In our previous study, ${ }^{25}$ the LPR position reaches its maximum within $5 \mathrm{~min}$, but the corresponding maximal absorbance increases slowly, over $15 \mathrm{~min}$. Since the observed absorbance increase corresponds to the volume increase, ${ }^{27,28}$ it is logical to accept the fact that the corresponding LPR blue shift occurs while the deposition is in process, thus supporting the second explanation above. The overgrowth has also been demonstrated by the TEM images of NRs in present work. Therefore, the reasonable explanation to the spectral shift here lays in AR decrease caused by the alteration of deposition rate on the side facets and end facets of NRs. This explaination is further supported by the fact that gold NRs can overgrow to produce octahedral particles enclosed entirely by $\{111\}$ facets at ends either through using excess ascorbic acid (AA) during NR growth ${ }^{29}$ or excess gold precursor. $^{30}$

Because the gold atoms are deposited only from the solvated gold salt, the most feasible pathway for controlling the AR adjustment is to deplete the gold salt in solution. The solvated gold salt is in form of $\mathrm{AuBr}_{2}{ }^{-}$due to the much higher bromide ion concentration. ${ }^{13,31,32}$ The depletion can be performed in two ways: either to $\mathrm{Au} 3+\left(\mathrm{AuBr}_{4}^{-}\right)$by oxidation or to $\mathrm{Au} 0$ (atom) by reduction. Oxidation depletion is impossible because of the etching of as-synthesized gold NRs by Au3 + that occurs when strong oxidant is added to the NRs solution. ${ }^{32}$ Also, it does not seem feasible to inhibit the deposition via reduction because this will inevitably result in the formation of gold atoms that promote NR growth. However, the preparation protocol of seeds for gold NRs sheds some light on this question, where a strong reducer, $\mathrm{NaBH}_{4}$, has been used to react with chloroauric acid, resulting in the formation of seeds, which are much smaller than the NRs. Moreover, $\mathrm{NaBH}_{4}$ has been utilized widely in gold cluster synthesis as a reducer. ${ }^{33-35}$ Interestingly, unlike the gradual growth of NRs, the cluster formation is usually rapid (occurring within several seconds) because of the strong reducibility of $\mathrm{NaBH}_{4}$. Another difference worth pointing out is that the seeds with small size $(<4 \mathrm{~nm})$ prefer to diffuse in solution and are almost impossible to be precipitated using centrifugation, while NRs can be easily precipitated from solution through centrifugation. ${ }^{36}$ These special features allow us to inhibit the deposition by introducing a competition process for precursor depletion using $\mathrm{NaBH}_{4}$ as a strong reducer that will form gold clusters, which can then be easily separated from the NRs.

\section{RESULTS AND DISCUSSION}

Following this strategy, experiments have been carried out by introducing $\mathrm{NaBH}_{4}$ into the reaction system whereas the evolution of $\mathrm{AR}$ was monitored using real-time absorption spectrum. Experimental details are given in the following section. The usual seed-mediated growth procedure reported previously has been used here. ${ }^{12,14}$ Note that to exclude the possibility that the reaction inhibition is due to AA depletion, we applied a ratio of $[\mathrm{AA}]:\left[\mathrm{HAuCl}_{4}\right]=1.5$ in the present synthesis, where the amount of ascorbic acid is sufficient to reduce all of the gold salt into gold atoms according to the stoichiometry of the reagents. ${ }^{37}$ Under this condition, a blue shift for LPR from 830 to $690 \mathrm{~nm}$ during the experimental time window (30 min) was observed, as shown in Figure 1A, which
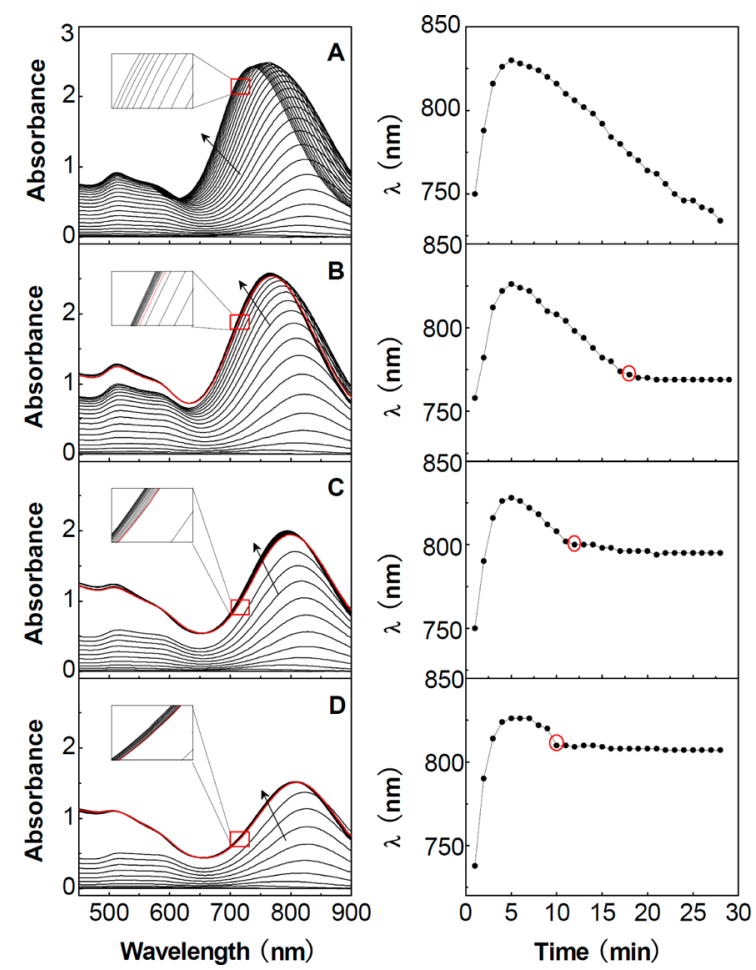

Figure 1. Left panel: evolution of absorption spectra of growth solutions. (A) Control sample without $\mathrm{NaBH}_{4}$ addition. (B-D) Samples with $\mathrm{NaBH}_{4}$ addition at different time points corresponding to the absorption peak position at 750,800 , and $820 \mathrm{~nm}$, respectively. Note that the first spectrum taken after $\mathrm{NaBH}_{4}$ addition is depicted in red line. For each panel, the inset shows the zoomed spectral profile. The arrows indicate the direction of evolution. Right panel: corresponding absorption peak position as function of time.

shows the possibility of LPR tuning within this wavelength range. Furthermore, in the inhibition experiment, excessive $\mathrm{NaBH}_{4}$ was added in order to make sure that all the residual gold salt could be reduced. In Figure $1 \mathrm{~B}-\mathrm{D}$, three representative results of spectral evolution, where $\mathrm{NaBH}_{4}$ solution was added at different time point, are shown, and the first spectrum just after $\mathrm{NaBH}_{4}$ addition is highlighted in red color. As expected, the spectral profile variations, including intensity and peak position of LPR, stop almost immediately upon $\mathrm{NaBH}_{4}$ addition in all three cases. The peak positions extracted from the absorption spectra are given on the right panel of Figure 1 as function of time, and the remarkable plateaus after $\mathrm{NaBH}_{4}$ addition (data point in red circle) indicate an effective suppression of the growth process. These results support the assumption that $\mathrm{NaBH}_{4}$, as an inhibitor, can be used to control the AR adjustment through completely consuming the gold precursor in the growth system.

Because the $\mathrm{NaBH}_{4}$ addition leads to an abrupt absorbance increase at shorter wavelength region $(<550 \mathrm{~nm})$, as shown in Figure $1 \mathrm{~B}-\mathrm{D}$, the influence of the addition of inhibitor on the 
final product quality should be taken into account. Assuming that the absorbance increase is attributed to the formation of the gold cluster, an effective approach to separate NRs from the ensemble mixture is critical. Conventional centrifugation has been employed herein to purify the samples, although there are some other approaches for isolating NRs from spherical particles, for example shape-dependent sedimentation. ${ }^{38}$ After centrifugation $(10000 \mathrm{rpm} \times 10 \mathrm{~min})$, dark-red sediments were obtained from the four samples corresponding to those in Figure 1. The sediments were washed twice and redispersed into water for spectral measurement. Figure 2I shows the
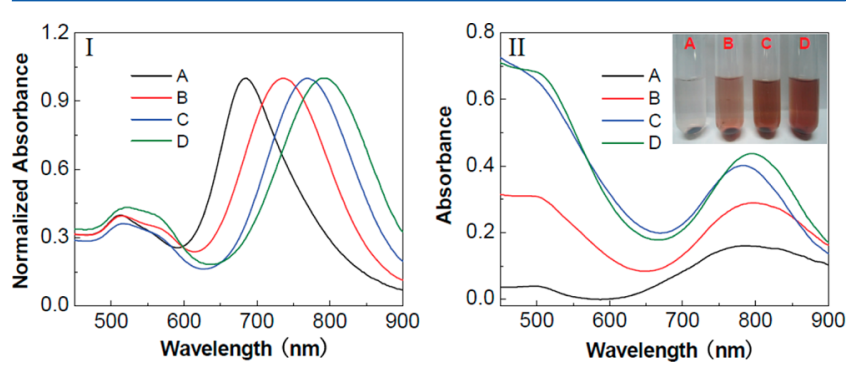

Figure 2. Absorption spectra of (I) the gold NRs and (II) the corresponding supernatant solutions after centrifugation. All curves correspond to the samples presented in Figure 1. The inset in II shows the samples after the first centrifugation.

spectra taken from the sediments, and spectral profiles typical for the gold NRs are observed for both the control sample (A) and three tested samples (B-D), indicating the existence of gold NRs. To confirm the results, we took TEM images (Figure 3 ) of the produced NRs; the results show that both the sample from normal growth procedure and those suffering an inhibition are dominated by rod-shaped particles, proving the purity of NRs.

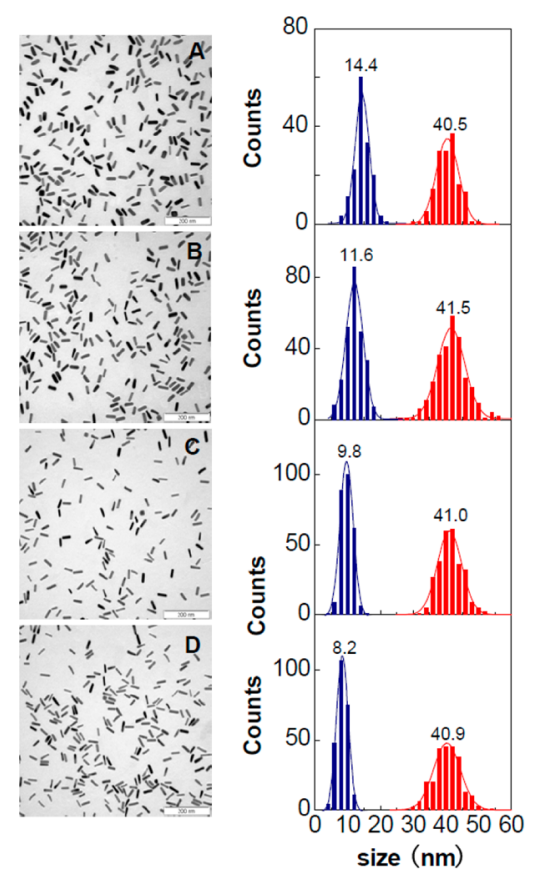

Figure 3. Left panel: TEM images of gold NRs corresponding to the samples in Figure 2A. Right panel: histograms of width (blue) and length (red) of gold NRs and the corresponding Gaussian fit results.
Furthermore, supernatants after the first centrifugation were collected, and absorption spectra were taken. As shown in Figure 2II, in the cases of the inhibited samples (B-D), the brown supernatants show significant absorption at the shorter wavelength, which is characteristic of gold clusters, ${ }^{14,35,39}$ indicating the presence of cluster in supernatants. On the other hand, due to the absence of clusters, the supernatant of control sample (A) is colorless and shows weak absorption, especially at the shorter wavelength range. Note that the observed absorption band similar to that of NRs at the longer wavelength in all supernatants should be ascribed to the residual gold NRs with small size, which have not been precipitated. No further centrifugation has been performed to collect this residual components and increase purity of the final product (with more uniform size distribution).

In addition, the TEM images were used for the size measurements, where for each sample 200 particles have been measured in order to obtain the size distribution. Figure 3 gives the histogram of width and length of each sample. The average lengths of four samples are around $41 \mathrm{~nm}$, whereas the widths vary with mean values of $14.4,11.6,9.8$, and $8.2 \mathrm{~nm}$ for samples $\mathrm{A}, \mathrm{B}, \mathrm{C}$, and $\mathrm{D}$, respectively. With the evidence of constant length but different widths, it is reasonable to accept the growth mechanism such that there should be a critical point, before which NRs exhibit a higher growth rate along the long axis than along the short axis. After this critical point, the growth rate along both axes tend to be the same. i.e. performing a nonselective deposition. The nonselective deposition on a rod-shaped particle surface leads to the same increase rate for width and length leading to a gradual decrease of the aspect ratio and a blue-shifted LPR. The growth interrupt also opens up the possibility to observe the morphology of the NRs at different stages, and to investigate the growth mechanism.

Although the addition of $\mathrm{NaBH}_{4}$ does inhibit the evolution of $\mathrm{AR}$, it also leads to decrease in the yield in term of gold salt, which is partially transferred into gold clusters. Here the yield should be proportional to the particle volume, because the same amount of seed has been used in all cases. According to the measured average size shown in Figure 3, the approximate volumes are $6592,4383,3091$, and $2158 \mathrm{~nm}^{3}$ for samples of $A$, $B, C$, and $D$, respectively. It is supposed that sample $A$, in which no cluster generates, has a yield of $100 \%$, the yield in B, C, and $\mathrm{D}$ can be calculated as $66.5,46.9$, and $32.7 \%$, respectively. Apparently, the case of higher AR corresponds to a lower yield. Our recent experiment showed that through altering the seed amount, the AR could also be tuned effectively, but with full utilization of gold salt. Furthermore, if the particle length and width at the critical point mentioned above is known, the morphology evolution during the whole growth process could be accurately predicted according to the initial reactant concentration. Detailed experimental investigation and modeling is underway and will be reported elsewhere.

On the other hand, we have tried to inhibit the deposition by depleting the reducer AA using strong oxidant. Herein, two oxidants, $\mathrm{H}_{2} \mathrm{O}_{2}$ and chloroauric acid, which will not introduce chemical contamination to growth system, were employed. Reactions between them and AA have been reported previously. ${ }^{14,19,40}$ Inhibiting experiments were conducted by adding either $\mathrm{H}_{2} \mathrm{O}_{2}$ or chloroauric acid solution into the growth system. Continuous blue shift of LPR was observed in both cases, indicating the inability of the both compounds to act as inhibitors (see Figures S1 and S2 in Supporting Information). Further inspection of the mechanism revealed that both 
oxidants have the ability to etch the gold NRs selectively at ends, resulting in AR decrease and associated LPR blue shift. ${ }^{23,24}$ Therefore, the continuous blue shift observed here should be ascribed to either the continuous gold deposition or end-etching of gold NRs, caused by the existence of either residual AA or over dose inhibitor, respectively. As known, since the oxidization-reduction potential is strongly dependent on many factors such as solution condition and concentration, it is hard to find a suitable oxidant which has capability to selectively oxidize AA but keep gold NRs from etching. Also, the possible product contamination should be considered carefully. Therefore, the deposition inhibition via AA depletion does not seem feasible.

\section{CONCLUSIONS}

In conclusion, we present an effective approach to synthesize gold nanorods with desirable LPR, in which the controllable AR decrease has been successfully utilized to finely tune LPR. Normally, in the presence of gentle reducer, gold salt is reduced into gold atom for nanoparticle growth, in particular nonspherical particle growth. However, the inevitable overgrowth does result in a decrease in aspect ratio, which has been demonstrated to be the main reason for the observed low reappearance of LPR. Given excess strong reducer was added into the growth system, as depicted in Figure 4, the competitive

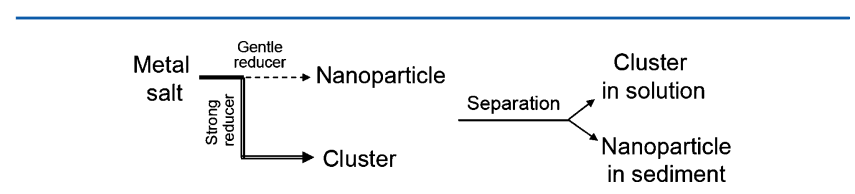

Figure 4. Schematic diagram of protocol for the growth inhibition by competitive reaction.

consumption of gold precursor leads to a sudden termination of the atom deposition on NRs. The efficient growth inhibition makes it possible to obtain gold NRs with any desired longitudinal plasmon resonance (presently in the $690-830 \mathrm{~nm}$ spectral range), and we believe that using an online monitor, for example, a fiber optical spectrometer, this approach could be extended to produce gold NRs in large quantity. Additionally, through stopping the particle growth at any stage, it also provides an opportunity to explore the crystallization mechanism of gold NRs and other nonspherical metal nanoparticles in solution.

\section{EXPERIMENTAL SECTION}

Materials. Chloroauric acid $\left(\mathrm{HAuCl}_{4}\right)$, ascorbic acid (AA), sodium borohydride $\left(\mathrm{NaBH}_{4}\right)$, and silver nitrate $\left(\mathrm{AgNO}_{3}\right)$ were purchased from Aldrich. Cetyltrimethylammonium bromide (CTAB) was purchased from Alfar. All reagents were used as received. Deionized water (>18.0 M $\Omega$ ) from a Millipore water purification system was used for all experiments.

Growth of Gold Nanorods. For seed preparation, $0.15 \mathrm{~mL}$ of icecold $10 \mathrm{mM} \mathrm{NaBH}_{4}$ was injected into $2 \mathrm{~mL}$ of rapidly stirred solution of $0.25 \mathrm{mM} \mathrm{HAuCl}_{4}$ in $0.1 \mathrm{M} \mathrm{CTAB}$. The pale brown solution was diluted 2 times and left for at least $2 \mathrm{~h}$ before use. A typical NRs synthesis involves a $2.0 \mathrm{~mL}$ aqueous solution consisting of $0.1 \mathrm{M}$ CTAB prepared in a $1 \mathrm{~cm}$ quartz optical cell. Also, $50 \mu \mathrm{L}$ of $20 \mathrm{mM}$ $\mathrm{HAuCl}_{4}$ solution, $15 \mu \mathrm{L}$ of $10 \mathrm{mM} \mathrm{AgNO}$, and $30 \mu \mathrm{L} 50 \mathrm{mM} \mathrm{AA}$ were added to the cell in turn followed by gentle mixing. AA amount is determined by the $[\mathrm{AA}]:\left[\mathrm{AuCl}_{4}\right]=1.5$ ratio. Finally, $10 \mu \mathrm{L}$ of the seed solution was injected into the mixture to initiate the growth of gold nanorods.
Growth Inhibition. To stop the growth process, $20 \mu \mathrm{L}$ of $\mathrm{NaBH}_{4}$ solution ( $50 \mathrm{mM}$ freshly made with ice-cold nanopure water) was injected into the mixture under stirring. A magnetic stirer was used to ensure complete mixing in the process of spectral recording ( $\sim 30 \mathrm{~s})$. Note that the amount of $\mathrm{NaBH}_{4}(1 \mu \mathrm{mol})$ applied is stoichiometrically sufficient to reduce all of the gold salt $(1 \mu \mathrm{mol})$ in the solution. In the case of ascorbic acid depletion, $\mathrm{NaBH}_{4}$ solution was replaced with 20 $\mu \mathrm{L}$ of hydrogen peroxide $(0.1 \mathrm{M})$ or $20 \mu \mathrm{L} \mathrm{HAuCl}_{4}(20 \mathrm{mM})$ solution.

Measurement. Absorption spectra in the $450-950 \mathrm{~nm}$ wavelength range were recorded with a Hitachi $3310 \mathrm{UV}$-vis spectrophotometer at room temperature using quartz cell of $10 \mathrm{~mm}$ path length. An automatically repetitive measurement has been carried out to record the spectral variation over time, and one spectrum was recorded per minute with a scan speed of $1200 \mathrm{~nm} / \mathrm{min}$. TEM images were acquired with a JEOL JEM-1011 transmission electron microscope. The samples for TEM were centrifuged $(10000 \mathrm{rpm} \times 10 \mathrm{~min})$ twice to remove the surfactant, and redispersed into water.

\section{ASSOCIATED CONTENT}

Supporting Information

Additional figures. This material is available free of charge via the Internet at http://pubs.acs.org/.

\section{AUTHOR INFORMATION}

\section{Corresponding Author}

*E-mail: wangxf@iccas.ac.cn.

\section{Notes}

The authors declare no competing financial interest.

\section{ACKNOWLEDGMENTS}

The authors acknowledge the financial support by the National Science Foundation of China (21003139). S.V: thanks CAS for support from the CAS Research Fellowship for International Young Researchers.

\section{REFERENCES}

(1) Xia, Y.; Xiong, Y.; Lim, B.; Skrabalak, S. E. Angew. Chem., Int. Ed. 2009, 48, 60-103.

(2) Huang, X.; Neretina, S.; El-Sayed, M. A. Adv. Mater. 2009, 21, $4880-4910$

(3) Huang, X.; El-Sayed, I. H.; Qian, W.; El-Sayed, M. A. J. Am. Chem. Soc. 2006, 128, 2115-2120.

(4) Zhou, W.; Shao, J.; Jin, Q.; Wei, Q.; Tangb, J.; Ji, J. Chem. Commun. 2010, 46, 1479-1481.

(5) Kuo, W. S.; Chang, C. N.; Chang, Y. T.; Yeh, C.-S. Chem. Commun. 2009, 4853-4855.

(6) Jang, B.; Park, J. Y.; Tung, C. H.; Kim, I. H.; Choi, Y. Nano 2011, 5, 1086-1094.

(7) Zhao, T.; Wu, H.; Yao, S. Q.; Xu, Q.-H.; Xu, G. Q. Langmuir 2010, 26, 14937-14942.

(8) Kuo, W.-S.; Chang, C.-N.; Chang, Y.-T.; Yang, M.-H.; Chien, Y.H.; Chen, S.-J.; Yeh, C.-S. Angew. Chem., Int. Ed. 2010, 49, 2711-2715.

(9) Xu, L.; Liu, Y.; Chen, Z.; Li, W.; Liu, Y.; Wang, L.; Liu, Y.; Wu, X.; Ji, Y.; Zhao, Y.; Ma, L.; Shao, Y.; Chen, C. Nano Lett. 2012, 12, 2003-2012.

(10) Durr, N. J.; Larson, T.; Smith, D. K.; Korgel, B. A.; Sokolov, K.; Ben-Yakar, A. Nano Lett. 2007, 7, 941-945.

(11) Zande, B. M. I. v. d.; Koper, G. J. M.; Lekkerkerker, H. N. W. J. Phys. Chem. B 1999, 103, 5754-5760.

(12) Jana, N. R.; Gearheart, L.; Murphy, C. J. Adv. Mater. 2001, 13, 1389-1393.

(13) Miranda, O. R.; Dollahon, N. R.; Ahmad, T. S. Cryst. Growth Des. 2006, 6, 2747-2753.

(14) Nikoobakht, B.; El-Sayed, M. A. Chem. Mater. 2003, 15, 19571962.

(15) Murazawa, N.; Ueno, K.; Mizeikis, V.; Juodkazis, S.; Misawa, H. J. Phys. Chem. C 2009, 113, 1147-1149. 
(16) Seo, S. S.; Wang, X.; Murray, D. Ionics 2009, 15, 67-71.

(17) Gou, L.; Murphy, C. J. Chem. Mater. 2005, 17, 3368-3472.

(18) Yong, K.-T.; Sahoo, Y.; Swihart, M. T.; Schneeberger, P. M.;

Prasad, P. N. Top. Catal. 2008, 47, 49-60.

(19) Sau, T. K.; Murphy, C. J. Langmuir 2004, 20, 6414-6420.

(20) Gole, A.; Murphy, C. J. Chem. Mater. 2004, 16, 3633-3640.

(21) Bullen, C.; Zijlstra, P.; Bakker, E.; Gu, M.; Raston, C. Cryst. Growth Des. 2011, 11, 3375-3380.

(22) Perez-Juste, J.; M.Liz-Marzan, L.; Carnie, S.; Chan, D. Y. C.; Mulvaney, P. Adv. Funct. Mater. 2004, 14, 571-579.

(23) Tsung, C.-K.; Kou, X.; Shi, Q.; Zhang, J.; Yeung, M. H.; Stucky, Wang, J.; Stuchy, G. D. J. Am. Chem. Soc. 2006, 128, 5352-5353.

(24) Ni, W.; Chen, H.; Su, J.; Sun, Z.; Wang, J.; Wu, H. J. Am. Chem. Soc. 2010, 132, 4806-4814.

(25) Wang, Y. Y.; Li, B. X.; Vdovic, S.; Wang, X. F.; Xia, A. D. Chinese J. Chem. Phys. 2012, 25, 135-141.

(26) Henkel, A.; Schubert, O; Plech, A.; Sonnichsen, C. J. Phys. Chem. C 2009, 113, 10390-10394.

(27) Orendorff, C. J.; Murphy, C. J. J. Phys. Chem. B 2006, 110, 3990-3994.

(28) Kooij, E. S.; Poelsema, B. Phys. Chem. Chem. Phys. 2006, 8, $3349-3357$.

(29) Xiang, Y.; Wu, X.; Liu, D.; Feng, L.; Zhang, K.; Chu, W.; Zhou, W.; Xie, S. J. Phys. Chem. C 2008, 112, 3203-3208.

(30) Carbó-Argibay, E.; Rodríguez-González, B.; Pacifico, J.; Pastoriza-Santos, I.; Pérez-Juste, J.; Liz-Marzán, L. M. Angew. Chem., Int. Ed. 2007, 46, 8983-8987.

(31) Lin, Z. J.; Chen, X. M.; Cai, Z. M.; Oyama, M.; Chen, X.; Wang, X. R. Cryst. Growth Des. 2008, 8, 863-868.

(32) Rodrıuez-Fernández, J.; Pérez-Juste, J.; Mulvaney, P.; LizMarzán, L. M. J. Phys. Chem. B 2005, 109, 14257-14261.

(33) Zhu, M.; Lanni, E.; Garg, N.; Bier, M. E.; Jin, R. J. Am. Chem. Soc. 2008, 130, 1138-1139.

(34) Negishi, Y.; Chaki, N. K.; Shichibu, Y.; Whetten, R. L.; Tsukuda, T. J. Am. Chem. Soc. 2007, 129, 11322-11323.

(35) Zhu, M.; Qian, H.; Jin, R. J. Am. Chem. Soc. 2009, 131, 72207221.

(36) Zheng, J.; Petty, J. T.; Dickson, R. M. J. Am. Chem. Soc. 2003, 125, 7780-7781.

(37) Gou, L.; Murphy, C. J. Chem. Mater. 2005, 17, 3668-3672.

(38) Park, K.; Koerner, H.; Vaia, R. A. Nano Lett. 2010, 10, 14331439.

(39) Ramakrishna, G.; Varnavski, O.; Kim, J.; Lee, D.; Goodson, T. J. Am. Chem. Soc. 2008, 130, 5032-5033.

(40) Uzu, T.; Sasaki, S. Org. Lett. 2007, 9, 4383-7386. 